\title{
DEBATES AND REVIEWS
}

The Journal of Language Contact was since the first volume meant for being a platform of discussion and did already include a section in which reviews of current publications on language contact and related disciplines were published. By following this initial attempt $J L C$ will upon now include a new section Debates. The purpose is to offer a mean of scientific dispute and to engage, again, debates on theoretical, epistemological or empirical issues in our respective academic field.

Articles published in this section do thus either discuss the implications of central hypothesis of a specific publication in its wider context, or deliberately initiate a topic pertinent to the overall of JLC without referring to a specific publication. Such contributions may be formulated as polemics - which play a incontestable role in the construction of scientific knowledge - they must be, however, seriously and carefully reasoned and should not at all include personal affronts ad hominem.

The Journal of Language Contact will thus contain the two following sections:

1) A Reviews section, in which reviews of current publications continue to be published like before (between 3500 and 9000 characters in length).

2) A Debates section, in which longer and more detailed texts are published (between 3500 and 70000 characters in length). 
Journal of Language Contact - VARIA 3 (2010)

www. jlc-journal.org 